\title{
a7 Nicotinic Acetylcholine Receptor Agonist PNU-282987 Ameliorates Cognitive Impairment Induced by Chronic Intermittent Hypoxia
}

\author{
Hui Shen \\ Yanling Meng \\ Dan Liu \\ Zheng Qin \\ Hong Huang \\ Lei Pan \\ Wei Wang \\ Jian Kang
}

Institute of Respiratory Disease, The First Hospital of China Medical

University, Shenyang, People's Republic of China
Correspondence: Wei Wang Institute of Respiratory Disease, The First Hospital of China Medical University, 155 Nanjing North Street, Heping District,

Shenyang, Liaoning, I I000I, People's

Republic of China

$\mathrm{Tel}+86 \quad 15242071600$

Fax +862483282002

Email wwbycmu@126.com
Purpose: Cognitive impairment is an important complication of obstructive sleep apnea (OSA). Chronic intermittent hypoxia $(\mathrm{CIH})$, the main pathophysiological characteristics of OSA, is closely related to cognitive dysfunction and may be mediated by alpha-7 nicotinic acetylcholine receptors $(\alpha 7 \mathrm{nAChR})$. This study investigated the effects and clarified the mechanisms of $\alpha 7 \mathrm{nAChR}$ on the cognitive function of mice with $\mathrm{CIH}$.

Methods: Thirty CD-1 mice were randomly divided into room air (RA), CIH-2 weeks (CIH2W), and CIH-4 weeks (CIH4W) groups. Cognitive function was evaluated by novel object recognition (NOR) and Morris water maze (MWM) tests after exposure. Then, 104 CD-1 mice were exposed to $\mathrm{CIH}$ for 4 weeks and randomly divided into four groups: CIH4W (control), with dimethyl sulfoxide (DMSO) (sham), with $\alpha 7 \mathrm{nAChR}$-specific agonist PNU282987 (PNU), and with $\alpha 7 n$ AChR-specific inhibitor methyllycaconitine and PNU-282987 (MLA+PNU). In addition to the evaluation of cognitive function, apoptotic bodies in the hippocampus were detected by terminal deoxynucleotidyl transferase dUTP nick end labeling (TUNEL) staining, changes in p-CREB and BDNF were detected by immunohistochemistry, while those of ERK1/2, CREB, PGC-1 $\alpha$, FNDC5, and BDNF were detected by Western blotting in the hippocampal tissues of the mice.

Results: Compared to the $\mathrm{CIH} 2 \mathrm{~W}$ and RA groups, the CIH4W group showed cognitive dysfunction in the NOR and MWM tests. The changes in cognitive dysfunction were alleviated by PNU-282987; furthermore, MLA pretreatment offset the effect. In hippocampal tissues, TUNEL assays showed decreased apoptotic cells, immunohistochemical staining showed increased expressions of p-CREB and BDNF. The expression levels of p-ERK1/2/ t-ERK1/2, p-CREB/t-CREB, PGC-1 $\alpha$, FNDC5, and BDNF were increased after PNU282987 injection.

Conclusion: Four weeks of $\mathrm{CIH}$ caused cognitive dysfunction in mice. Activating $\alpha 7 \mathrm{nAChR}$ might ameliorate this dysfunction by upregulating the ERK1/2/CREB signaling pathway; enhancing PGC-1 $\alpha$, FNDC5, and BDNF expression levels; and reducing cell apoptosis in the hippocampal tissue of mice.

Keywords: hypoxemia, cognitive dysfunction, nicotinic acetylcholinergic receptor alpha7 subunit, CREB, BDNF, obstructive sleep apnea

\section{Introduction}

Cognitive impairment refers to the pathological process associated with the abnormal processing of advanced brain intelligence related to learning, memory, and thinking judgment. In severe cases, cognitive impairment can develop into Alzheimer's disease or even dementia, which can seriously affect patient quality 
of life and increase the medical burden. Recent studies have shown that obstructive sleep apnea (OSA) is closely related to cognitive dysfunction. ${ }^{1-3}$ Repeated complete or incomplete collapse of the upper respiratory tract during sleep, the main manifestation of OSA, can cause pathological and physiological changes such as repeated decrease of oxygen saturation at night, sleep fragmentation, and enhanced sympathetic autonomous activity. Therefore, OSA can affect the function of multiple systems including the cardiovascular, neuropsychiatric, and endocrine systems. ${ }^{4}$ As a common complication of OSA, cognitive impairment has attracted attention in recent years. The effects of OSA on cognitive function include decreased attention and alertness, impaired memory and learning ability, imbalanced psychomotor function, disturbed emotional regulation, and decreased executive function., ${ }^{1,5}$ A study following 298 older women without dementia for 2 years found that patients with sleep-disordered breathing were more likely to develop mild cognitive impairment or dementia. ${ }^{5}$ Chronic intermittent hypoxia $(\mathrm{CIH})$, the main pathophysiological characteristic of OSA, is currently believed to be associated with the occurrence of cognitive dysfunction in patients with OSA. However, the specific mechanism remains unclear.

The central cholinergic system plays an important role in the regulation of cognitive function, including the formation of learning and memory. A decrease in the number of cholinergic neurons is related to Alzheimer's disease (AD) and dementia. ${ }^{6}$ The excitability and release of neurotransmitters of cholinergic neurons are regulated by the nicotinic acetylcholinergic receptor (nAChR). Animal studies have shown that $\mathrm{nAChR}$ in the hippocampus is related to the regulation of working memory. Blocking $\mathrm{nAChR}$ in the ventral or dorsal hippocampus caused an increase in the number of work errors in the radial arm maze task. ${ }^{7,8}$ In the central nervous system, the most expressed nAChR subtypes are $\alpha 4 \beta 2$ and $\alpha 7$, among which $\alpha 7 \mathrm{nAChR}$ is widely distributed in the I and VI layers of the cerebral cortex, CA1, CA3, and dentate gyrus (DG) of the hippocampus. ${ }^{9} \alpha 7 \mathrm{nAChR}$ activation can mediate the transduction of ERK1/2 and CREB, the key component of learning and memory formation, in the MAPKs pathway. ${ }^{10-12}$ Wang et al found that $\mathrm{CIH}$ reduced CREB levels of neurons in the CA1 region of the hippocampus in Sprague Dawley (SD) rats. ${ }^{13}$ Thus, we speculated that $\mathrm{CIH}$ could induce cognitive impairment through an $\alpha 7 \mathrm{nAchR}-$ related mechanism. This study aimed to identify the protective role of $\alpha 7 \mathrm{nAChR}$ in cognitive function in a mouse model of $\mathrm{CIH}$ and to explore the underlying mechanisms.

\section{Materials and Methods \\ Animals}

Four-week-old male CD-1 mice were purchased from Beijing Vital River Laboratory Animal Technologies Co., Ltd. The mice were provided a standard chow diet and water ad libitum and housed in clean polypropylene cages, with a 12-hour light and dark cycle (lights on from 8:00 am to $8: 00 \mathrm{pm})$. The room temperature was maintained at $25 \pm 2^{\circ} \mathrm{C}$. All experiments were approved by the ethics committee of China Medical University and conducted following the regulations of the laboratory animal department of China Medical University and the State Science and Technology Commission about the care and use of laboratory animals.

\section{Experimental Protocol}

The two parts of this experiment included a total of 134 mice. In the first part, the mice were divided into three groups: the room air (RA, $\mathrm{n}=10), \mathrm{CIH}-2$ weeks $(\mathrm{CIH} 2 \mathrm{~W}$, $\mathrm{n}=10$ ), and $\mathrm{CIH}-4$ weeks $(\mathrm{CIH} 4 \mathrm{~W}, \mathrm{n}=10)$ groups. When the CIH4W group mice had been treated with $\mathrm{CIH}$ for 2 weeks, the $\mathrm{CIH} 2 \mathrm{~W}$ group mice began to be put into the hypoxic chamber. At the end of the exposure, every mouse underwent novel object recognition (NOR) and Morris water maze (MWM) tests. In the second part of the experiment, 104 mice were randomly divided into four groups: CIH-4 weeks (CIH4W, n=26), CIH-4 weeks with DMSO (sham, $\mathrm{n}=26$ ), CIH-4 weeks with $\alpha 7 \mathrm{nAChR}$-specific agonist PNU-282987 (PNU, n=26), and CIH-4 weeks with a7nAChR-specific inhibitor methyllycaconitine and PNU-282987 (MLA+PNU, n=26) groups. At the end of the exposure, 10 mice from each group underwent NOR and MWM tests, another 8 mice from each group were prepared for terminal deoxynucleotidyl transferase (TdT)mediated dUTP nick-end labeling (TUNEL) and immunohistochemical staining, and the remaining 8 mice from each group were prepared for Western blotting.

\section{$\mathrm{ClH}$ Protocol}

All mice were placed in chambers (Oxycycler model A48XOV, BioSherix, USA) for 8 hours every day from 8:00 am to 4:00 pm. The mice were randomly divided into $\mathrm{RA}$ and $\mathrm{CIH}$ group. The $\mathrm{CIH}$ group mice were placed in 
chambers with a $\mathrm{CIH}$ profile consisting of 30 seconds of normal oxygen $\left(21 \% \mathrm{O}_{2}\right), 30$ seconds of hypoxia $\left(6 \% \mathrm{O}_{2}\right)$, and 30 seconds for the oxygen concentration to rise and fall, 40 cycles/hr. The mice in the RA group were subjected to chambers with room air in identical experimental conditions. The oxygen concentration in the chambers was continuously measured by an oxygen concentration monitor and adjusted by a computerized system by modulating the nitrogen and oxygen gas outlets.

\section{Drug Administration}

Mice in the PNU group received $5 \mathrm{mg} / \mathrm{kg}$ of PNU-282987 (sigma, USA) intraperitoneally. The MLA+PNU group received $5 \mathrm{mg} / \mathrm{kg}$ of MLA (Abcam, UK) intraperitoneally 15 minutes before receiving $5 \mathrm{mg} / \mathrm{kg}$ of PNU-282987 intraperitoneally. Mice in the sham group received $2 \%$ DMSO intraperitoneally. All treatments were administered $30 \mathrm{~min}$ before hypoxic exposure from the 26th day of $\mathrm{CIH}$.

\section{NOR Tests}

NOR tests were performed in a box $(50 \mathrm{~cm}$ in length $\times 50 \mathrm{~cm}$ in width $\times 50 \mathrm{~cm}$ in height) with surrounding walls and bottom painted black. On the first day of the experiment, every mouse was placed into the test box containing no objects and allowed to acclimate for 5 minutes. Stool and urine were removed and the box and surfaces were sprayed with $70 \%$ ethanol to avoid lingering odors before the next mouse was placed in the box. On the second day, two identical objects (old object) were placed in the test box and each mouse was placed into the test box for 5 minutes. On the third day, one old object and one different new object (new object) were placed in the test box. Each mouse was placed into the box for $5 \mathrm{~min}$, with a camera recording the lengths of time the mouse spent exploring the new and old objects. The mouse was identified as exploring when its nose tip was less than $1 \mathrm{~cm}$ from the object or if it touched the object with its front paws or nose. The discrimination index was used to compare and analyze the duration differences in the exploration of the old and new objects. ${ }^{14,15}$ The calculation formula was as follows: Discrimination index $=$ (new object exploration time (seconds) - old object exploration time (seconds))/ total object exploration time (seconds) $\times 100 \%$.

\section{MWM Tests}

The MWM tests were conducted after the NOR tests. This test utilized a circular tank 1.5 meters in diameter with a rough-surface platform at $25 \mathrm{~cm}$ in height and $6 \mathrm{~cm}$ in diameter. The pool was divided into four quadrants by two mutually perpendicular virtual lines. A platform was located in the middle of one of the four quadrants, $0.5-1 \mathrm{~cm}$ below the surface of the water during the first 5 training days. The water and room temperatures were controlled at $22 \pm 1^{\circ} \mathrm{C}$ and $25 \pm 1^{\circ} \mathrm{C}$, respectively. Ethovision XT software was used for data recording and analysis, with the whole pool and surrounding reference objects unchanged during training. The training was started after $\mathrm{CIH}$ every day. On the first day (day 0), the mice were put into the water and allowed to swim freely until they found the platform and allowed to rest on the platform for more than $15 \mathrm{~s}$ before being removed. If a mouse could not find the platform within $120 \mathrm{~s}$, it was guided to the platform and kept on the platform for more than $15 \mathrm{~s}$. Each mouse entered the MWM from two directions. The mice were put into the water facing the wall of the pond when a trial began. The acquisition trials were conducted from days 1 to 4 . Each mouse was put into the pool from three different quadrants every day. The swimming speed and the time to find the platform (escape latency) were calculated for each trial. On day 5, as the probe trial, the platform was removed and the mice were put into the water for $120 \mathrm{~s}$. The number of times that each mouse crossed the original platform (platform crossings) was calculated.

\section{Tissue Processing}

At the end of $\mathrm{CIH}$, mice ( $\mathrm{n}=16$ from each group in the second part of the experiment) were anesthetized with pentobarbital sodium. The heads of some mice were cut off quickly; the skulls were then quickly peeled off on ice to separate the hippocampus, which was placed in liquid nitrogen and stored at $-80^{\circ} \mathrm{C}$. The other mice were transcardially perfused with $4 \%$ paraformaldehyde solution in $0.1 \mathrm{M}$ phosphate buffer (PBS, pH7.4). Their heads were then cut off and the brain tissue removed and placed in $4 \%$ paraformaldehyde to fix the cells. The tissue blocks were dehydrated with gradient ethanol (concentrations ranging from $70 \%$ to $100 \%$ ), transparently treated with xylene, and then embedded in paraffin. Finally, the paraffin blocks were cut into $4-\mu \mathrm{m}$ thick slices and placed on polylysine-coated slides for TUNEL and immunohistochemistry assays.

\section{TUNEL Staining}

To observe the neuroprotective effect of PNU-282987, TUNEL assays were used to detect apoptotic cells. After deparaffinization in dimethylbenzene, the brain tissue sections were rehydrated in gradient ethanol (concentrations ranging from $100 \%$ to $70 \%$ ), washed with pure water and 
PBS, incubated with proteinase $\mathrm{K}$ for $10 \mathrm{~min}$ before incubation in the TUNEL reaction mixture (beyotime, China) for 1 hour in the dark at $37^{\circ} \mathrm{C}$. Finally, the sections were added with antifade mounting medium with 4',6-diamidino-2-phenylindole (DAPI). With an inverted fluorescent microscope (Leica, Germany), TUNEL positive cells were observed at $520 \mathrm{~nm}$ of green fluorescence, nuclei were observed at $460 \mathrm{~nm}$ of blue fluorescence. Image $\mathrm{J}$ software was used to calculate the number of apoptotic cells in the hippocampus.

\section{Immunohistochemical Staining}

After rehydration, the brain tissue sections were immersed in $3 \% \mathrm{H}_{2} \mathrm{O}_{2}$ solution for $30 \mathrm{~min}$ at room temperature to block endogenous peroxidase activity. The tissue sections were then subjected to citrate buffer $(10 \mathrm{mM}, \mathrm{pH} 6.0)$ for antigen retrieval in a microwave oven. Normal goat serum was added for another 30 minutes at room temperature to block nonspecific binding, followed by incubation with rabbit anti phosphorylated CREB (p-CREB) antibody (1:200, CST, USA) or rabbit recombinant anti-brain-derived neurotrophic factor (BDNF) antibody (1:400, Abcam, Cambridge) overnight at $4^{\circ} \mathrm{C}$. After washing with PBS for $15 \mathrm{~min}$, the tissue sections were incubated with the secondary antibody for 1 hour. The excess secondary antibody was then removed and a streptavidin peroxidase complex was placed on the tissue sections for $20 \mathrm{~min}$, followed by 3,3-diaminobenzidine (DAB) for several minutes for dyeing. The chromogenic condition was observed under the microscope and the chromogenic time was recorded while the target protein was brownish-yellow. The tissue sections were then counterstained with hematoxylin, differentiated with alcohol and hydrochloric acid, and flushed with water. No brown structures were observed in the blank control sections. Image $\mathrm{J}$ analysis software was used for quantification.

\section{Western Blotting}

The frozen tissues were homogenized in ice-cold radioimmunoprecipitation assay (RIPA) buffer containing phosphatase inhibitor (beyotime, China) for $30 \mathrm{~min}$. Then, after centrifugation at $4^{\circ} \mathrm{C}$ and $12,000 \mathrm{rpm}$ for 30 minutes, the supernatant was extracted and the protein concentration was calculated by bicinchoninic acid (BCA) protein assay reagent (Thermo, USA). Equal amounts of protein (50 $\mu \mathrm{g})$ were boiled with loading buffer as sample, then separated on $10-12.5 \%$ sodium dodecyl sulfate-polyacrylamide gel electrophoresis (SDS-PAGE) gels through electrophoresis, transferred onto polyvinylidene fluoride membrane, and incubated with primary antibody overnight at $4^{\circ} \mathrm{C}$. Anti- p44/42 MAPK (ERK1/2) (Thr202/Tyr204) (1:1000, CST, USA), anti-phospho-p44/42 MAPK (p-ERK1/2) (Thr202/ Tyr204) (1:1000, CST, USA), anti-p-CREB (Ser133) (1:1000, CST, USA), anti-CREB (Ser133) (1:1000, CST, USA), rabbit recombinant monoclonal anti-fibronectin type III domain containing protein 5 (FNDC5) (1:1000, Abcam, UK), rabbit anti-peroxisome proliferator-activated receptor $\gamma$ (PPAR $\gamma)$ coactivator-1 $\alpha$ (PGC-1 $\alpha)(1: 1000$, Abcam, UK), rabbit recombinant monoclonal anti-BDNF (1:1000, Abcam, UK), mouse monoclonal anti- $\beta$-actin (1:1000, Zhongshan Golden Bridge Biotechnology, Beijing) and antirabbit/mouse IgG HRP-linked secondary antibodies (1:5000, Abcam, UK) were used. Proteins were visualized by Enhanced Chemiluminescence kit (Thermo, USA) and the intensities of the bands were quantified by optical density methods using Image J software (NIH).

\section{Statistical Analysis}

All data were expressed as means $\pm \mathrm{SD}$. The data were analyzed using one-way analysis of variance (ANOVA) followed by least significant difference (LSD) post hoc (equal variances) or Dunnett's T3 (equal variances not assumed) tests. $\mathrm{P}<0.05$ was considered to indicate a significant difference.

\section{Results \\ Effects of $\mathrm{ClH}$ on NOR Tests in Mice}

We observed no significant difference in the total object exploration time among the three groups (Figure 1A). Compared to the RA and $\mathrm{CIH} 2 \mathrm{~W}$ groups, the CIH4W group had a significantly lower discrimination index (Figure 1B).

\section{Effects of $\mathrm{ClH}$ on MWM Tests in Mice}

We observed no significant difference in swimming speeds of the mice during the 4 days of acquisition trials, suggesting that $\mathrm{CIH}$ did not affect their locomotor activity (Figure 1C). The mean escape latency gradually decreased with increased training trials (Figure 1D). Compared to the RA and CIH2W groups, the CIH4W group showed significantly longer escape latencies on days $1-4$, suggesting that 4 weeks instead of 2 weeks of CIH impair the learning and memory ability of mice. In the probe trial on day 5, compared to the RA group, the number of platform crossings was significantly reduced in the CIH4W group and not significantly different in the CIH2W group (Figure 1E). Significant differences were observed between the $\mathrm{CIH} 2 \mathrm{~W}$ and $\mathrm{CIH} 4 \mathrm{~W}$ groups, indicating that the duration of $\mathrm{CIH}$ treatment had different effects on behavioral tests reflecting the cognitive function of mice. 
A

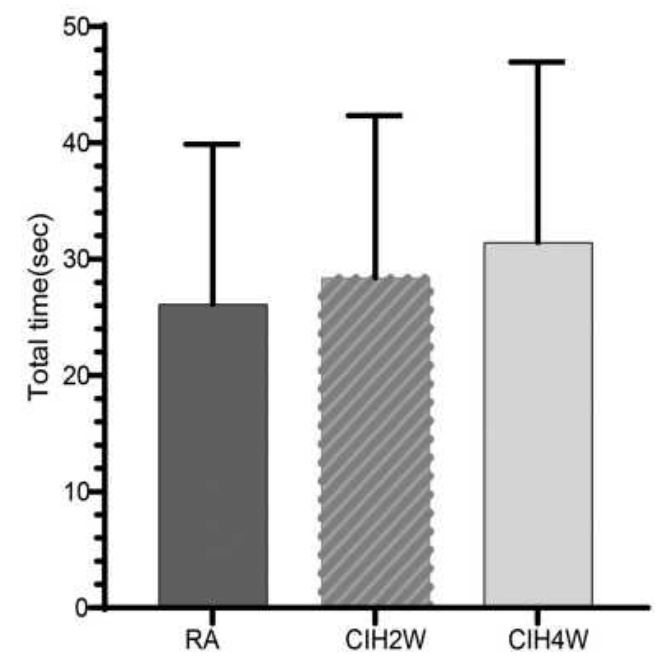

B

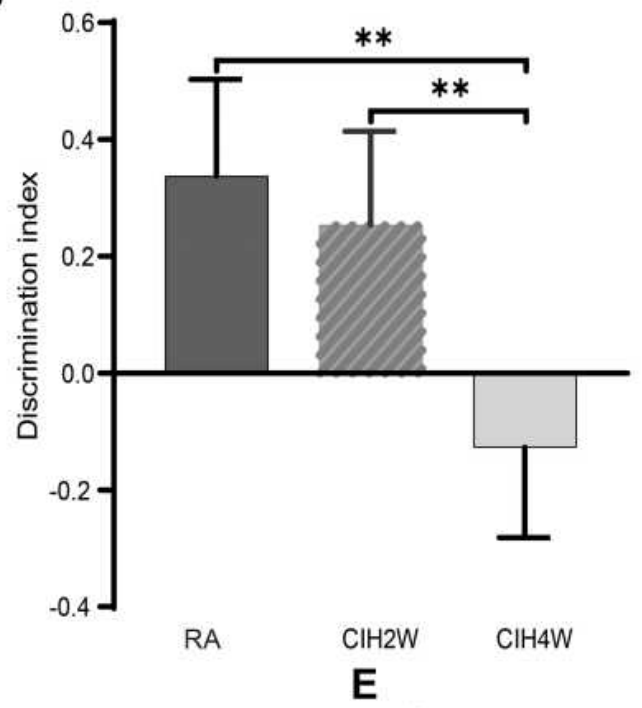

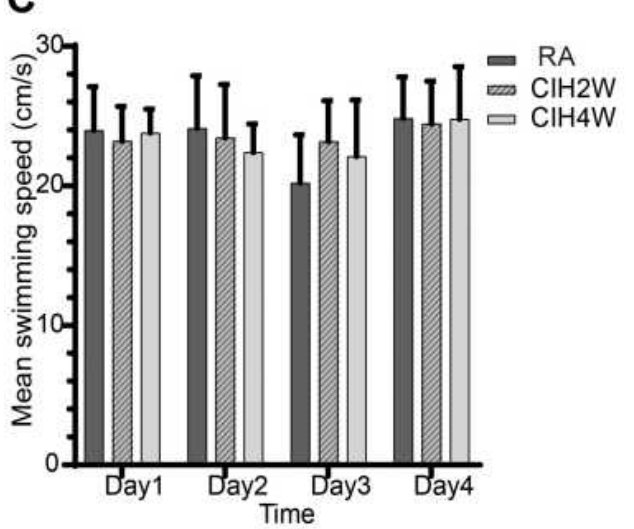

D
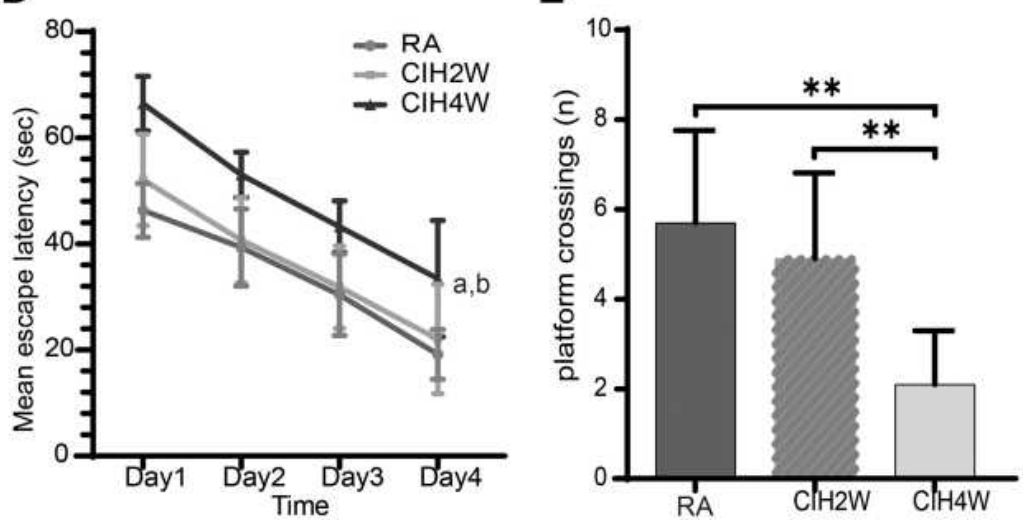

Figure I Comparisons of behavior test results among room air (RA), $\mathrm{ClH}-2$ weeks (ClH2W), and $\mathrm{ClH}-4$ weeks (ClH4W) groups. Comparisons among the three groups of total object exploration times $(\mathbf{A})$ and discrimination index $(\mathbf{B})$ in the NOR test, mean escape speed in the MWM test (C), mean daily escape latency in spatial acquisition trials on days I-4 in the MWM test (D), and the number of platform crossings in the probe test of the $\mathrm{MWM}$ on day 5 (E). ${ }^{* *} \mathrm{P}<0.0 \mathrm{I}$. ${ }^{\mathrm{a}} \mathrm{P}<0.05$ compared to the $\mathrm{ClH} 2 \mathrm{~W}$ group, ${ }^{\mathrm{b}} \mathrm{P}<0.05$ compared to the RA group.

Abbreviations: NOR, novel object recognition; MWM, Morris water maze.

\section{Effects of PNU-282987 on NOR Tests in Mice}

We observed no significant differences in the total object exploration times among the four groups (Figure 2A), indicating that the administration of agonist or inhibitor did not affect object exploration in mice. Compared to those in the CIH $(-0.141 \pm 0.125)$, sham $(-0.082 \pm 0.161)$, and the MLA+PNU $(-0.183 \pm 0.295)$ groups, the discrimination index of the PNU group $(0.180 \pm 0.105)$ was significantly increased (Figure 2B), suggesting that PNU could improve the memory ability of $\mathrm{CIH}$ mice.

\section{Effects of PNU-282987 on MWM Tests in Mice}

During the 4 training days, we observed no significant difference in the speed of mice swimming in the water (Figure
2C). The increased escape latency of the CIH4W group was alleviated by the administration of PNU-282987. When MLA was added, the escape latency increased again (Figure 2D). On the probe trial day, compared to $\mathrm{CIH}$, sham, and MLA+PNU groups, the PNU group showed an increased number of platform crossings (Figure 2E). These results suggested that $\alpha 7 \mathrm{nAChR}$ activation improved spatial learning and memory disorders in $\mathrm{CIH}$ mice.

\section{Effects of PNU-282987 on Apoptosis in the Hippocampus of $\mathrm{ClH}$ Mice}

To evaluate the neuroprotective effect of PNU-282987, we detected cell apoptosis in the hippocampus of $\mathrm{CIH}$ mice by TUNEL staining. As shown in Figure 3, TUNEL staining showed significantly decreased apoptotic cells in the PNU group compared to those in the $\mathrm{CIH}$ and sham groups. When 


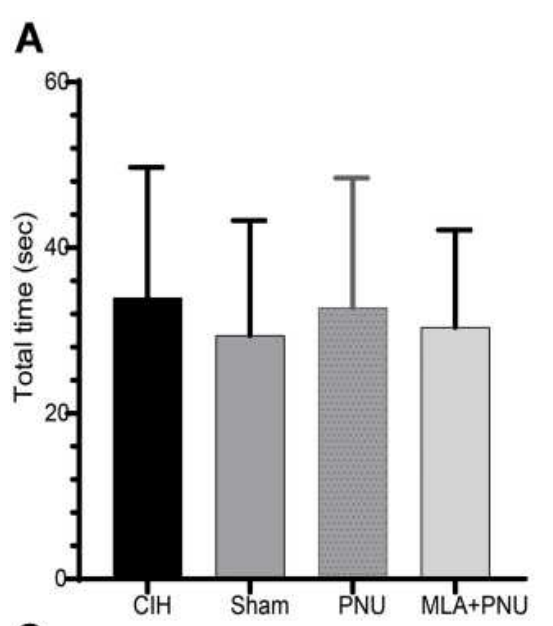

C

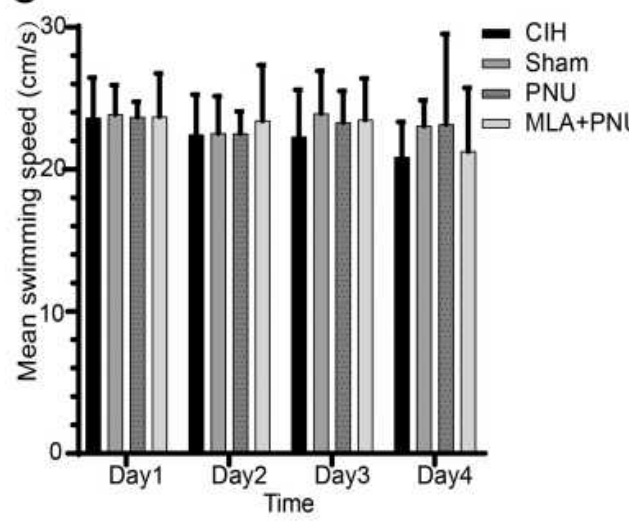

B



D

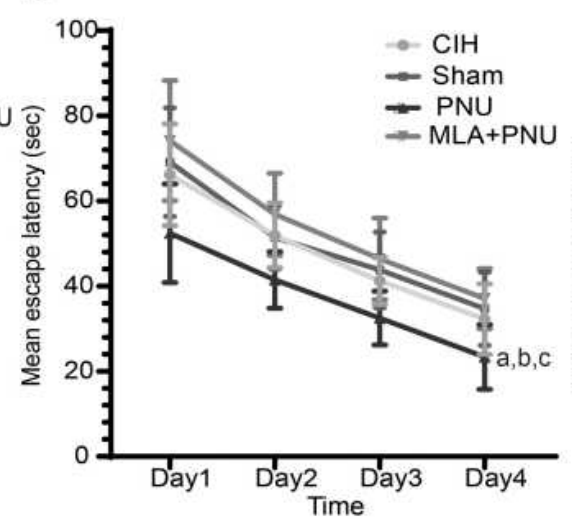

E



Figure 2 Comparisons of behavior test results among the ClH4W (control), with DMSO, with $\alpha 7 n A C h R$-specific agonist PNU-282987 (PNU), and with $\alpha 7 n A C h R-s p e c i f i c$ inhibitor methyllycaconitine and PNU-282987 (MLA+PNU) groups. Comparisons of total object exploration time (A) and discrimination index (B) in the NOR test, mean escape speed in the MWM (C), mean escape latency in spatial acquisition trials on days I-4 in the MWM (D), and the number of platform crossings in the probe test of the MWM on day $5(\mathbf{E})$. **P<0.01. ${ }^{\text {a }} \mathrm{P}<0.05$ compared to the $\mathrm{CIH}$ group, ${ }^{\mathrm{b}} \mathrm{P}<0.05$ compared to the sham group, ${ }^{\mathrm{C}} \mathrm{P}<0.05$ compared to the $\mathrm{MLA}+\mathrm{PNU}$ group.

Abbreviations: NOR, novel object recognition; MWM, Morris water maze.

A



DAPI
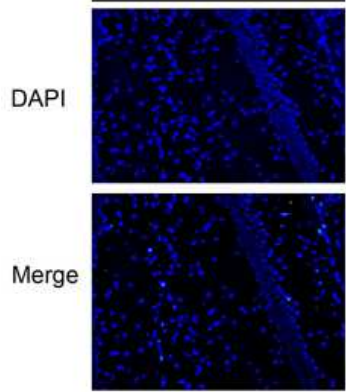

sham
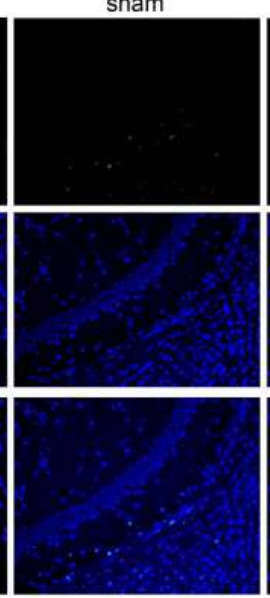

PNU
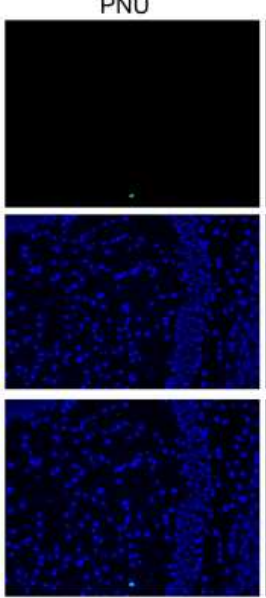

MLA+PNU



B

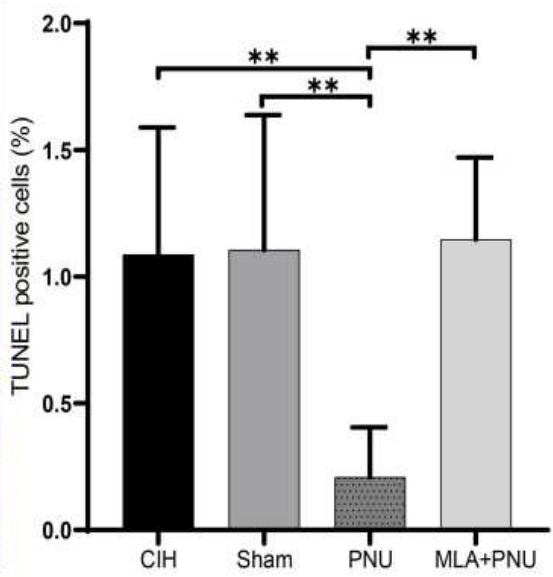

Figure 3 Effects of PNU-282987 on apoptosis in the hippocampus of mice. (A) Results of TUNEL staining in hippocampal tissue from each group. Scale bar = 200 $\mu$ m. (B) The index of TUNEL positive cells in hippocampus. ${ }^{* * P}<0.01$. TUNEL, terminal deoxynucleotidyl transferase-mediated dUTP nick-end labeling. 
MLA was added, the number of apoptotic cells did not decrease, suggesting that PNU-282987 had neuroprotective effect in $\mathrm{CIH}$ mice.

\section{$\alpha 7 n A c h R$ Activation Increases the Expressions of ERKI/2/CREB Signaling Pathway-Associated Proteins in the Hippocampus of $\mathrm{ClH}$ Mice}

To investigate the protective effects of PNU-282987, immunohistochemical staining was used to measure p-CREB expression in the hippocampal CA1, CA3, and DG regions, while Western blotting was used to measure the protein levels of key components of the ERK1/2/CREB pathway in the hippocampus. The immunohistochemical staining suggested that, compared to the $\mathrm{CIH}$ and sham groups, the expressions of p-CREB increased in the hippocampal CA1, CA3, and DG regions of the PNU group (Figure 4A). The levels of p-ERK1/2/t-ERK1/2 and p-CREB/t-CREB increased in the PNU group compared to those in the $\mathrm{CIH}$ and sham groups (Figure 4B-D). These results suggested that PNU-282987 activated the ERK1/2/CREB signaling pathway.

\section{$\alpha 7 n A c h R$ Activation Increases the Expression of Downstream PGC-I $\alpha /$ FNDC5/BDNF Pathway-Associated} Proteins in the Hippocampus of $\mathrm{ClH}$ Mice The present study subsequently evaluated BDNF expression level in the hippocampal CA1, CA3, and DG regions
A

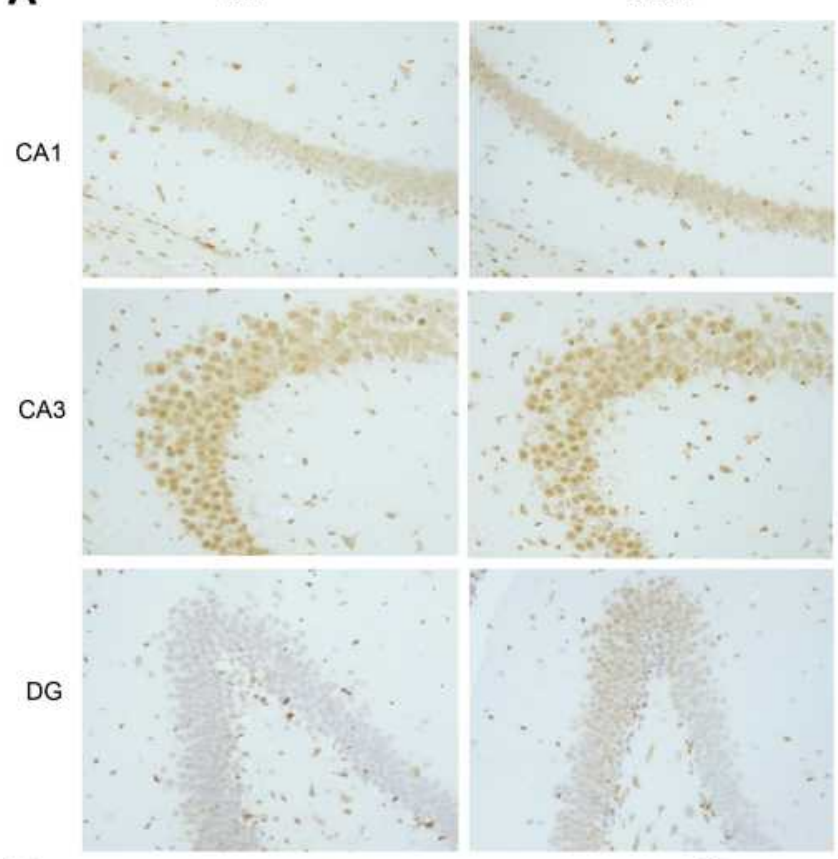

PNU
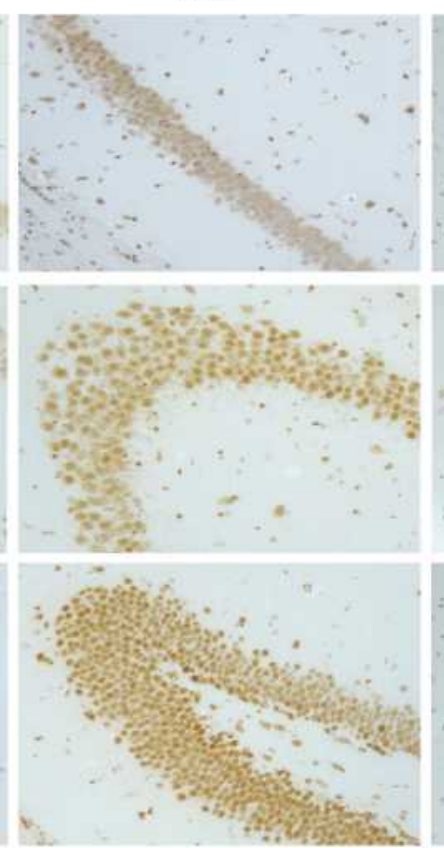

B

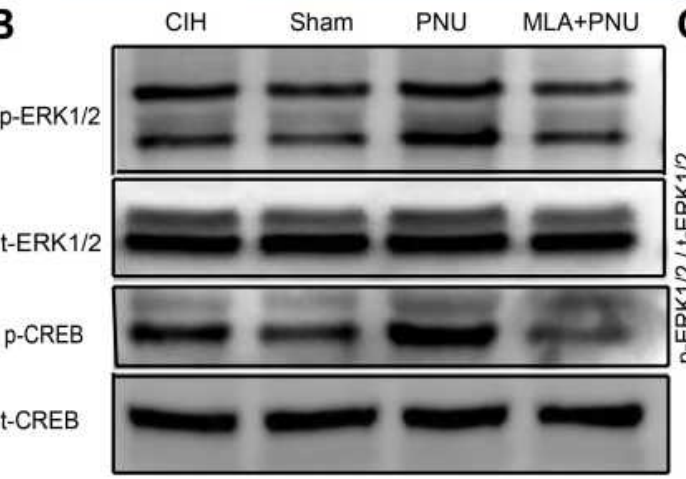

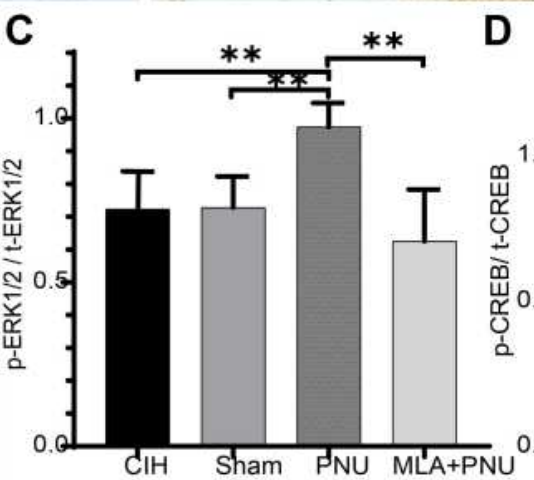

$M L A+P N U$
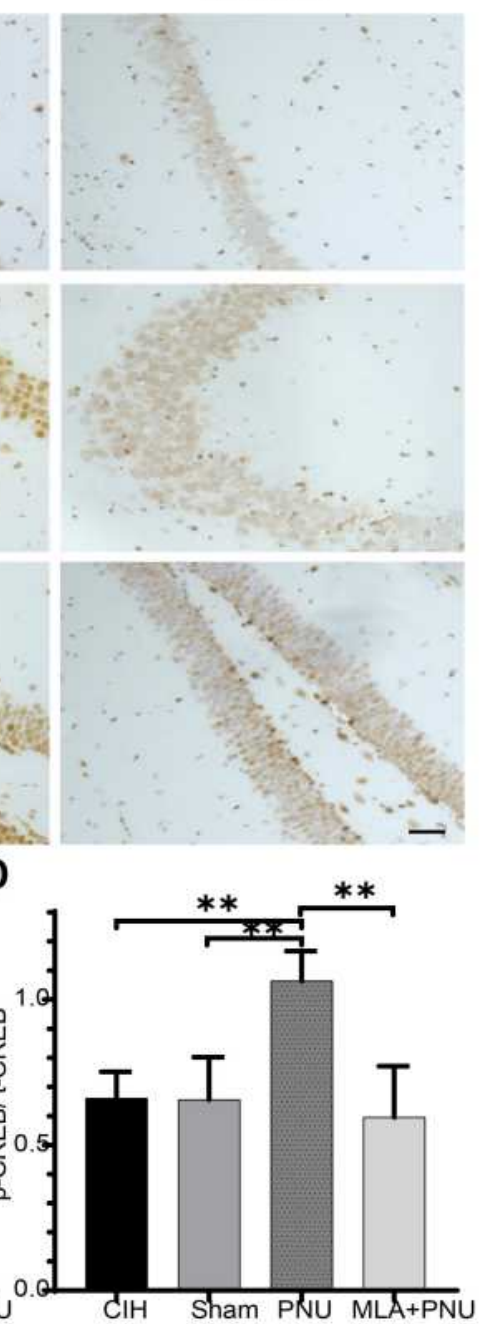

Figure 4 Effects of PNU-282987 on ERKI/2 and CREB expression in mice. (A) Results of immunohistochemical staining results for p-CREB in hippocampal tissue from each group. Scale bar $=50 \mu \mathrm{m}$. (B) Expression of ERKI/2/CREB pathway proteins in the hippocampal tissues of each group. (C) Comparisons of $\mathrm{p}$-ERKI/2/t-ERKI/2 in the hippocampal tissues of each group. (D) Comparisons of $\mathrm{p}$-CREB/t-CREB in the hippocampal tissues of each group. **p<0.0I. 
by immunohistochemical staining and the protein levels of PGC-1 $\alpha$, FNDC5, BDNF in the hippocampus by Western blotting. As shown in Figure 5A, immunohistochemical staining showed markedly increased BDNF expression after PNU-282987 injection. The results of Western blotting showed that significantly increased expressions of PGC-1 $\alpha$, FNDC5, and BDNF in the PNU group compared to those in the $\mathrm{CIH}$ and sham groups (Figure 5B-E). Although the expression levels of PGC-1 $\alpha$, FNDC5, and BDNF were decreased in the MLA+PNU group, the difference was not statistically significant. These results suggested the upregulation of PGC-1 $\alpha$, FNDC5, and BDNF expression by PNU-282987 treatment.

\section{Discussion}

$\mathrm{CIH}$, the main pathological characteristics of OSA, leads to pathological alterations in the central nervous system including decreased hippocampal synaptic plasticity, neurodegeneration, and apoptosis, which are closely associated with cognitive and memory impairment. ${ }^{16-18}$ In the present study, 4 weeks of $\mathrm{CIH}$ exposure caused cognitive dysfunction in mice, as assessed by NOR and MWM tests. Moreover, the exogenous intraperitoneal injection of the $\alpha 7 n$ AChR agonist PNU-282987 improved cognitive dysfunction, an effect offset by pretreatment of the $\alpha 7 \mathrm{nAChR}$-specific inhibitor MLA. These results indicated that these alterations were related to $\alpha 7 \mathrm{nAChR}$ and that $\alpha 7$ nAChR activation might improve $\mathrm{CIH}$-induced cognitive dysfunction. Further analysis showed that $\alpha 7 \mathrm{nAChR}$ regulated cognitive function through the ERK1/2/CREB pathway.

In 2001, Gozal et al established the CIH model of rats, observed impaired cognitive function of CIH-treated rats through MWM tests, and found that $\mathrm{CIH}$ for 14 days caused significant declines in spatial learning and memory ability. ${ }^{19}$ The results of the present study demonstrated decreased cognitive function of CD-1 mice, manifested as longer escape latency in MWM tests and reduced numbers of platform crossings in the exploration tests, in 4 rather than 2 weeks of $\mathrm{CIH}$. These results may differ from those of Gozal et al due to difference in hypoxic profiles, in which they used room air $\left(21 \% \mathrm{O}_{2}\right)$ and hypoxia $(10 \%$ $\mathrm{O}_{2}$ ) every 90 seconds, 12 hours a day, compared to room air $\left(21 \% \mathrm{O}_{2}\right)$ and hypoxia $\left(6 \% \mathrm{O}_{2}\right)$ every 30 seconds, 8 hours a day in the present study. Our experiment results were in consistent with previous researches. ${ }^{13,18}$ Wang et al found that 4 weeks of $\mathrm{CIH}$ treatment caused lower neurobehavioral performance in MWM tests in mice. ${ }^{13}$
Yin et al found that 30 days of $\mathrm{CIH}$ exposure caused neurocognitive dysfunction and neuron apoptosis in SD rats. ${ }^{18}$ The present study also evaluated the cognitive function of mice by NOR tests. The NOR test was first described by Ennaceur in 1988 to test the visual object recognition and memory ability of mice based on their natural curiosity. ${ }^{14}$ As expected, the present study observed a reduced discrimination index in the NOR test in $\mathrm{CIH}$-treated mice. $\mathrm{CIH}$ is characterized by hypoxiareoxygenation cycles, which can lead to intermittent cerebral hypoxia and even apoptosis of brain cells in mice, similar to cerebral infarction caused by ischemia and hypoxia. ${ }^{20}$ However, the specific mechanism of cognitive impairment induced by $\mathrm{CIH}$ remains unclear.

The neurotransmitter ACh regulates neuronal excitability through nAChRs that are widely distributed in the central nervous system and participate in various physiological responses, including anxiety and cognitive function. $^{21,22} \mathrm{CIH}$-induced cognitive dysfunction was related to decreased choline acetyltransferase (CHAT) levels in the forebrains of SD rats. ${ }^{23}$ Bancroft and Levin injected the selective $\alpha 7 \mathrm{nAchR}$ inhibitor MLA and $\alpha 4 \beta 2 \mathrm{nAchR}$ inhibitor $\mathrm{DH} \beta \mathrm{E}$ into the dorsal hippocampus of SD rat, which resulted in significantly increased working memory and reference memory errors in 16-arm radial water maze tests. ${ }^{7,8}$ Agnieszka et al showed that the $\alpha 7$ nAChR-positive allosteric modulator galantamine combined with memantine improved the discrimination index of rats in NOR tests. ${ }^{24}$ Sun et al reported the intravenous injection of an $\alpha 7 \mathrm{nAChR}$ agonist 6 hours after middle cerebral artery embolization (MCAO) in SD rats results in reduced cerebral infarct volume and improved neurological function in Bederson, rolling cylinder, and ladder rung walking tests 24 hours after MCAO. ${ }^{25}$ Hijioka et al treated mice with intracerebral hemorrhage (ICH) with different doses of the $\alpha 7 \mathrm{nAChR}$ agonist PNU-282987, reporting similar results between $3 \mathrm{mg} / \mathrm{kg}$ and $10 \mathrm{mg} / \mathrm{kg}$ doses both of which reduced the accumulation of activated microglia and macrophages in the perihematomal region and significantly increased the number of surviving neurons in the central and peripheral regions of hematoma at 3 days after $\mathrm{ICH}^{26}$ These results suggested the neuroprotective effect of $\alpha 7 \mathrm{nAChR}$ agonists. Using a $\mathrm{CIH}$ model, we observed that intraperitoneal injection of PNU-282987 improved the discrimination index of mice in NOR tests, and significantly shortened escape latency and increased the number of platform crossings in MWM tests, which further demonstrated that $\alpha 7 \mathrm{nAChR}$ agonists participated 
A

$\mathrm{ClH}$

Sham

PNU

$M L A+P N U$
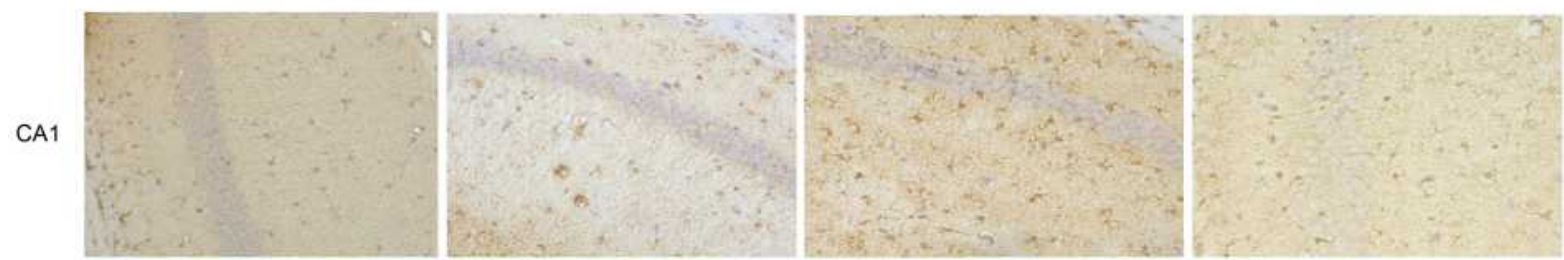

$\mathrm{CA} 3$
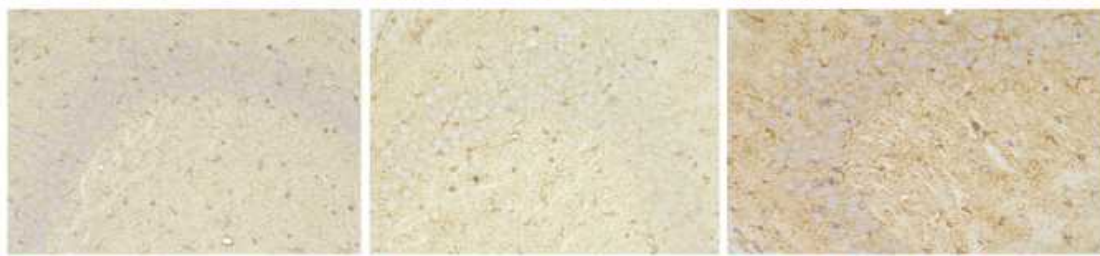

DG
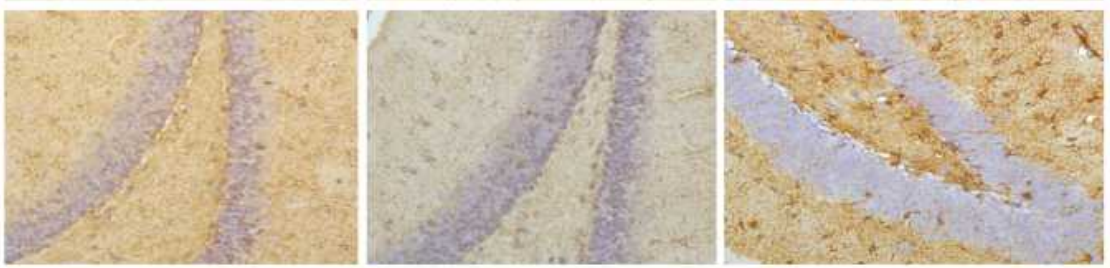

B



D
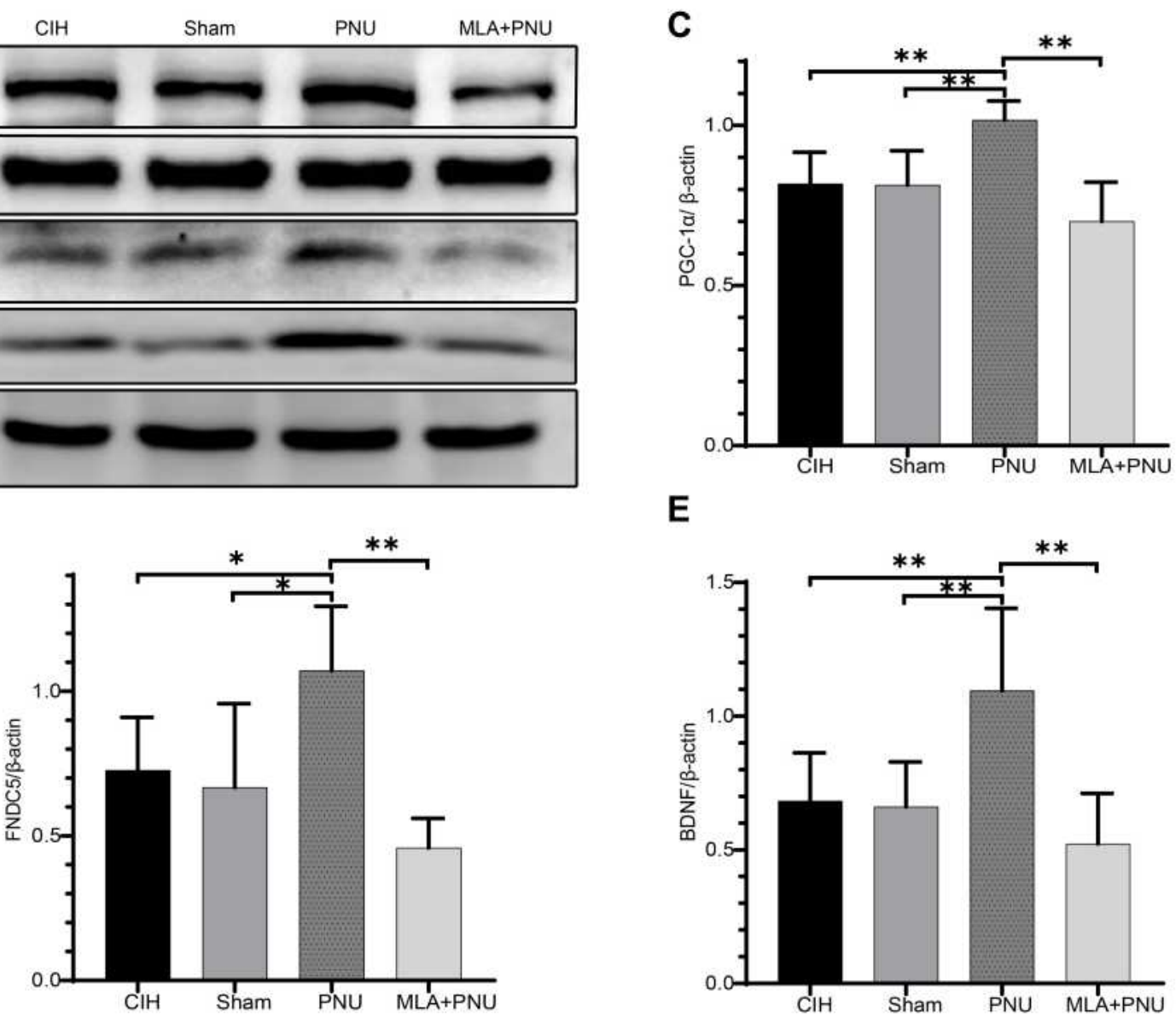

E

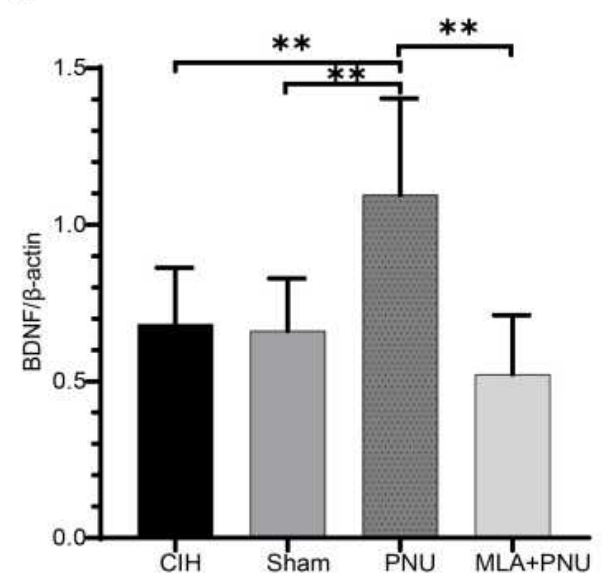

Figure 5 Effects of PNU-282987 on PGC-I $\alpha$, FNDC5, and BDNF protein expression in the hippocampus of mice. (A) BDNF levels in each group. Scale bar $=50 \mu m$. (B) PGC-I $\alpha$, FNDC5, and BDNF expression in each group. (C) Comparisons of PGC-I $\alpha$ expression in each group. (D) Comparisons of FNDC5 expression in each group. (E) Comparisons of BDNF expression in each group. ${ }^{*} \mathrm{p}<0.05$, $* * \mathrm{p}<0.01$. 
in the CIH-induced cognitive impairment in mice. Furthermore, no protective effect against cognitive dysfunction was observed for the intraperitoneal administration of PNU-282987 15 minutes after the intraperitoneal injection of MLA. These findings again verified that $\alpha 7 \mathrm{nAChR}$ activation could improve $\mathrm{CIH}$-induced cognitive dysfunction in mice. We detected cell apoptosis in the hippocampus of CIH mice by TUNEL staining, found that the number of apoptotic cells was significantly decreased in the PNU group compared to those in the CIH and sham groups. However, the number did not decrease when MLA was pretreated, suggesting that PNU-282987 had neuroprotective effect in $\mathrm{CIH}$ mice.

By administrating transgenic (Tg) 6799 (5XFAD) mice with cotinine, a choline metabolite, Grizzell et al observed improved discrimination index of mice in NOR tests, as well as increased p-CREB expression. ${ }^{27}$ PNU-282987 upregulated p-ERK1/2, p-CREB, and BDNF expression and decreased the number of BrdU-positive cells in the DG region of the hippocampus in CaMKIV null mice. ${ }^{28}$ In CIH-treated mice, Wang et al observed that neurocognitive dysfunction caused by $\mathrm{CIH}$ was associated with ERK1/2 phosphorylation and CREB expression. ${ }^{13}$ The results of the present study showed increased $p$-ERK1/2 and p-CREB expression in the hippocampus of mice after injection of the $\alpha 7 \mathrm{nAChR}$-specific agonist PNU-282987 compared to that in the CIH group. However, the expression levels of $\mathrm{p}$-ERK1/2 and p-CREB did not increase when the $\alpha 7 \mathrm{nAchR}$-specific inhibitor MLA was administered before PNU-282987, suggesting that $\alpha 7 n A C h R$ activation improved $\mathrm{CIH}$-induced cognitive dysfunction through the ERK1/2/CREB signaling pathway.

CREB regulates PGC- $1 \alpha$ and BDNF expression. ${ }^{29,30}$ PGC-1 $\alpha$, a member of the PGC-1 protein family, is an interaction factor of nuclear receptor transcription factor $\operatorname{PPAR} \gamma$, which can protect against neuronal damage. ${ }^{31}$ PGC-1 $\alpha$ regulates BDNF expression through FNDC5, thereby enhancing LTP and improving learning and memory ability in mice. ${ }^{32}$ BDNF is highly expressed in the brain and plays an important role in synapse development. It can also regulate neural behavior by regulating synaptic plasticity in the brain. Xie et al found that $\mathrm{CIH}$ reduced BDNF expression in the hippocampus of mice, leading to deficits in memory formation; this cognitive impairment was prevented by the microinjection of BDNF into the brain of CIH-treated mice. ${ }^{33}$ Wang and colleagues reported that pretreatment of chronic intermittent hypobaric hypoxia could ameliorate ischemia/reperfusion-induced cognitive dysfunction in MWM and step-down avoidance tests, and increase neuron density in the hippocampal CA1 subfield by activating the ERK1/2/CREB/BDNF signaling pathway. ${ }^{30}$ PGC-1 $\alpha /$ FNDC5/BDNF is a key pathway for neuroprotection and cognitive function. ${ }^{34-36}$ Liu et al reported that acute hypobaric hypoxia caused cognitive impairment by reducing PGC-1 $\alpha$, FNDC5, and BNDF expression, while upregulating PGC- $1 \alpha /$ FNDC5/BNDF pathway levels prevented cognitive impairment. ${ }^{34}$ Furthermore, the results of the present study showed significantly increased PGC-1 $\alpha$, FNDC5, and BDNF expression in the hippocampus of CIH-treated mice after $\alpha 7 \mathrm{nAChR}$ activation. However, PGC-1 $\alpha$, FNDC5, and BDNF expression did not increase when MLA was administered before PNU-282987, suggesting that $\alpha 7 \mathrm{nAChR}$ activation may improve $\mathrm{CIH}$-induced cognitive dysfunction through regulation of PGC-1 $\alpha$, FNDC5, and BDNF levels.

The limitation of this study was that $\alpha 7 \mathrm{nAChR}$ expression was not directly detected; thus, it was not clear whether the $\alpha 7 \mathrm{nAChR}$-specific agonist PNU-282987 worked by activating receptor activity or increasing the number of receptors. However, we found that the $\mathrm{CIH}$ induced cognitive dysfunction improved after intraperitoneal injection of PNU-282987 and that pretreatment of MLA eliminated the effect, which verified the participation of $\alpha 7 \mathrm{nAChR}$ in the regulation of $\mathrm{CIH}$-induced cognitive dysfunction.

\section{Conclusion}

The results of our study showed that PNU-282987 could ameliorate $\mathrm{CIH}$-induced cognitive dysfunction by upregulating the ERK1/2/CREB signaling pathway, enhancing the expression of PGC-1 $\alpha$, FNDC5, and BDNF, and reducing cell apoptosis in the hippocampal tissue of mice. These results provide a basis for the study of the mechanism of cognitive dysfunction in patients with OSA and identify a new potential target for the treatment of cognitive dysfunction caused by OSA.

\section{Acknowledgments}

We would like to thank Editage for English language editing.

\section{Funding}

This work was financially supported by the Outstanding Talent Project of Liaoning Provincial Talent Work Leading Group (grant number XLYC1805002). 


\section{Disclosure}

The authors report no conflicts of interest in this work.

\section{References}

1. Zhou J, Camacho M, Tang X, Kushida CA. A review of neurocognitive function and obstructive sleep apnea with or without daytime sleepiness. Sleep Med. 2016;23:99-108. doi:10.1016/j.sleep.2016. 02.008

2. Naismith S, Duffy S, Cross N, et al. Nocturnal Hypoxemia Is Associated with Altered Parahippocampal Functional Brain Connectivity in Older Adults at Risk for Dementia. $J$ Alzheimer Dis. 2020;73(2):571-584. doi:10.3233/JAD-190747

3. Cross N, Memarian N, Duffy S, et al. Structural brain correlates of obstructive sleep apnoea in older adults at risk for dementia. Eur Respir J. 2018;52(1):1800740. doi:10.1183/13993003.00740-2018

4. Salman L, Shulman R, Cohen J. Obstructive Sleep Apnea, Hypertension, and Cardiovascular Risk: epidemiology, Pathophysiology, and Management. Curr Cardiol Rep. 2020;22 (2):6. doi:10.1007/s11886-020-1257-y

5. Yaffe K, Laffan AM, Harrison SL, et al. Sleep-disordered breathing, hypoxia, and risk of mild cognitive impairment and dementia in older women. JAMA. 2011;306(6):613-619. doi:10.1001/jama.2011.1115

6. Petrova T, Orellana C, Jelic V, et al. Cholinergic dysfunction, neurodegeneration, and amyloid-beta pathology in neurodegenerative diseases. Psychiatry Res Neuroimaging. 2020;302:111099. doi:10.1016/j.pscychresns.2020.111099

7. Bancroft A, Levin ED. Ventral hippocampal alpha4beta2 nicotinic receptors and chronic nicotine effects on memory. Neuropharmacology. 2000;39(13):2770-2778. doi:10.1016/S00283908(00)00099-X

8. Levin ED, Bradley A, Addy N, Sigurani N. Hippocampal alpha 7 and alpha 4 beta 2 nicotinic receptors and working memory. Neuroscience. 2002;109(4):757-765. doi:10.1016/S0306-4522(01)00538-3

9. Gotti C, Zoli M, Clementi F. Brain nicotinic acetylcholine receptors: native subtypes and their relevance. Trends Pharmacol Sci. 2006;27 (9):482-491. doi:10.1016/j.tips.2006.07.004

10. Ren K, Puig V, Papke RL, Itoh Y, Hughes JA, Meyer EM. Multiple calcium channels and kinases mediate alpha7 nicotinic receptor neuroprotection in PC12 cells. J Neurochem. 2005;94(4):926-933. doi:10.1111/j.1471-4159.2005.03223.x

11. Roman J, Ritzenthaler JD, Gilacosta A, Rivera HN, Roserpage S. Nicotine and fibronectin expression in lung fibroblasts: implications for tobacco-related lung tissue remodeling. THE FASEB Journal. 2004;18(12):1436-1438. doi:10.1096/fj.03-0826fje

12. Thomas GM, Huganir RL. MAPK cascade signalling and synaptic plasticity. Nat Rev Neurosci. 2004;5(3):173-183. doi:10.1038/nrn 1346

13. Wang J, Ming H, Chen R, et al. CIH-induced neurocognitive impairments are associated with hippocampal $\mathrm{Ca}(2+)$ overload, apoptosis, and dephosphorylation of ERK1/2 and CREB that are mediated by overactivation of NMDARs. Brain Res. 2015;1625:64-72. doi:10.1016/j.brainres.2015.08.012

14. Ennaceur A, Delacour J. A new one-trial test for neurobiological studies of memory in rats. 1: behavioral data. Behav Brain Res. 1988;31(1):47-59. doi:10.1016/0166-4328(88)90157-X

15. Hornick A, Schwaiger S, Rollinger J, Vo N, Prast H, Stuppner H. Extracts and constituents of Leontopodium alpinum enhance cholinergic transmission: brain $\mathrm{ACh}$ increasing and memory improving properties. Biochem Pharmacol. 2008;76(2):236-248. doi:10.1016/j. bcp.2008.04.015

16. Khuu MA, Pagan CM, Nallamothu $T$, et al. Intermittent Hypoxia Disrupts Adult Neurogenesis and Synaptic Plasticity in the Dentate Gyrus. J Neurosci. 2019;39(7):1320-1331. doi:10.1523/ JNEUROSCI.1359-18.2018
17. Zhou B, Li M, Cao X, et al. Phenylethanoid glycosides of Pedicularis muscicola Maxim ameliorate high altitude-induced memory impairment. Physiol Behav. 2016;157:39-46. doi:10.1016/j.physbeh. 2016.01.037

18. Yin X, Zhang X, Lv C, et al. Protocatechuic acid ameliorates neurocognitive functions impairment induced by chronic intermittent hypoxia. Sci Rep. 2015;5:14507. doi:10.1038/srep14507

19. Gozal D, Daniel JM, Dohanich GP. Behavioral and anatomical correlates of chronic episodic hypoxia during sleep in the rat. $J$ Neurosci. 2001;21(7):2442-2450. doi:10.1523/JNEUROSCI.2107-02442.2001

20. Cai X, Li X, Jin S, Liang D, Wen Z. Endoplasmic reticulum stress plays critical role in brain damage after chronic intermittent hypoxia in growing rats. Exp Neurol. 2014;257:148-156. doi:10.1016/j. expneurol.2014.04.029

21. Dineley K, Pandya A, Yakel J. Nicotinic ACh receptors as therapeutic targets in CNS disorders. Trends Pharmacol Sci. 2015;36 (2):96-108. doi:10.1016/j.tips.2014.12.002

22. Alzarea S, Rahman S. Alpha-7 nicotinic receptor allosteric modulator PNU120596 prevents lipopolysaccharide-induced anxiety, cognitive deficit and depression-like behaviors in mice. Behav Brain Res. 2019;366:19-28. doi:10.1016/j.bbr.2019.03.019

23. Row B, Kheirandish L, Cheng Y, Rowell P, Gozal D. Impaired spatial working memory and altered choline acetyltransferase (CHAT) immunoreactivity and nicotinic receptor binding in rats exposed to intermittent hypoxia during sleep. Behav Brain Res. 2007;177 (2):308-314. doi:10.1016/j.bbr.2006.11.028

24. Nikiforuk A, Potasiewicz A, Kos T, Popik P. The combination of memantine and galantamine improves cognition in rats: the synergistic role of the $\alpha 7$ nicotinic acetylcholine and NMDA receptors. Behav Brain Res. 2016;313:214-218. doi:10.1016/j.bbr.2016.07.023

25. Sun F, Jin K, Uteshev V. A type-II positive allosteric modulator of $\alpha 7$ nAChRs reduces brain injury and improves neurological function after focal cerebral ischemia in rats. PLoS One. 2013;8(8):e73581. doi:10.1371/journal.pone.0073581

26. Hijioka M, Matsushita H, Ishibashi H, Hisatsune A, Isohama Y, Katsuki H. alpha7 Nicotinic acetylcholine receptor agonist attenuates neuropathological changes associated with intracerebral hemorrhage in mice. Neuroscience. 2012;222:10-19. doi:10.1016/j.neuroscience. 2012.07.024

27. Alex GJ, Patel S, Barreto GE, Echeverria V. Cotinine improves visual recognition memory and decreases cortical Tau phosphorylation in the Tg6799 mice. Prog Neuropsychopharmacol Biol Psychiatry. 2017;78:75-81. doi:10.1016/j.pnpbp.2017.05.010

28. Moriguchi S, Inagaki R, Yi L, Shibata M, Sakagami H, Fukunaga K. Nicotine Rescues Depressive-like Behaviors via $\alpha 7$-type Nicotinic Acetylcholine Receptor Activation in CaMKIV Null Mice. Mol Neurobiol. 2020;57(12):4929-4940. doi:10.1007/s12035-020-02077-z

29. Zhong J, Dong W, Qin Y, et al. Roflupram exerts neuroprotection via activation of CREB/PGC-1 $\alpha$ signalling in experimental models of Parkinson's disease. Br J Pharmacol. 2020;177(10):2333-2350. doi:10.1111/bph.14983

30. Wang J, Zhang S, Ma H, et al. Chronic Intermittent Hypobaric Hypoxia Pretreatment Ameliorates Ischemia-Induced Cognitive Dysfunction Through Activation of ERK1/2-CREB-BDNF Pathway in Anesthetized Mice. Neurochem Res. 2017;42(2):501-512. doi:10.1007/s11064-016-2097-4

31. Knutti D, Kralli A. PGC-1 a versatile coactivator. Trends Endocrinol Metab. 2001;12(8):360-365. doi:10.1016/S1043-2760(01)00457-X

32. Wrann C, White J, Salogiannnis J, et al. Exercise induces hippocampal BDNF through a PGC-1 $\alpha$ /FNDC5 pathway. Cell Metab. 2013;18 (5):649-659. doi:10.1016/j.cmet.2013.09.008

33. Xie H, Leung K-L, Chen L, et al. Brain-derived neurotrophic factor rescues and prevents chronic intermittent hypoxia-induced impairment of hippocampal long-term synaptic plasticity. Neurobiol Dis. 2010;40(1):155-162. doi:10.1016/j.nbd.2010.05.020 
34. Liu P, Zou D, Yi L, et al. Quercetin ameliorates hypobaric hypoxiainduced memory impairment through mitochondrial and neuron function adaptation via the PGC-1 $\alpha$ pathway. Restor Neurol Neurosci. 2015;33(2):143-157. doi:10.3233/RNN-140446

35. Xia D, Huang X, Bi C, Mao L, Peng L, Qian H. PGC- $1 \alpha$ or FNDC5 Is Involved in Modulating the Effects of $A \beta$ Oligomers on Suppressing the Expression of BDNF, a Beneficial Factor for Inhibiting Neuronal Apoptosis, $A \beta$ Deposition and Cognitive Decline of APP/PS1 Tg Mice. Front Aging Neurosci. 2017;9:65. doi:10.3389/fnagi.2017.00065
36. Belviranlı M, Okudan N. Exercise training protects against aginginduced cognitive dysfunction via activation of the hippocampal PGC-1 $\alpha /$ FNDC5/BDNF Pathway. Neuromolecular Med. 2018;20 (3):386-400. doi:10.1007/s12017-018-8500-3

\section{Publish your work in this journal}

Nature and Science of Sleep is an international, peer-reviewed, open access journal covering all aspects of sleep science and sleep medicine, including the neurophysiology and functions of sleep, the genetics of sleep, sleep and society, biological rhythms, dreaming, sleep disorders and therapy, and strategies to optimize healthy sleep.
The manuscript management system is completely online and includes a very quick and fair peer-review system, which is all easy to use. Visit http://www.dovepress.com/testimonials.php to read real quotes from published authors. 